\title{
Geometric Theory of Stark Resonances in Multielectron Systems
}

\author{
I. M. Sigal* \\ Department of Mathematics, University of Toronto, Toronto, Canada M5S 1A1
}

\begin{abstract}
In this paper we consider a class of many-body systems in a weak homogeneous electric field. This class includes atoms and molecules with infinitely heavy nuclei. It follows from one of the results of this paper and a result of [S 3] that the bound states of such systems in the absence of electric field turn into resonances (which we call the Stark resonances) as soon as the electric field is switched on. (The stability part of this result was earlier proven in [HeSi] (see also [Hu 2]) under an assumption of dilation analyticity.) The main result of this paper is exponential bounds on the width (and therefore the lower exponential bounds on the life-time) of the Stark resonances. These bounds are given in terms of the Stark instanton action. In contrast to the usual (one body) action the latter is not entirely classical but incorporates certain quantum data (like ionization energies). The bounds give a partial generalization to the many electron case of the well-known Oppenheimer formula for the hydrogen.
\end{abstract}

\section{Table of Contents}

1. Introduction . . . . . . . . . . . . . . . . . . . . . . . . . . 288

2. Hamiltonian . . . . . . . . . . . . . . . . . . . . . . . 288

3. Instanton Metric . . . . . . . . . . . . . . . . . . . . . . . . 289

4. Energetically Forbidden Region . . . . . . . . . . . . . . . . . . . 291

5. Spectral Deformations . . . . . . . . . . . . . . . . . . . . . . . 293

6. Main Result. . . . . . . . . . . . . . . . . . . . . . . . . . . 296

7. Energy Inequalities . . . . . . . . . . . . . . . . . . . . . . . . 297

8. Hamiltonians $H_{f}^{(i)}$. . . . . . . . . . . . . . . . . . . . . . . . . . . . 298

9. Stability of Resonances. . . . . . . . . . . . . . . . . . . . . . . 300

10. Exponential Bounds on Eigenfunctions of $H_{f}^{0}$. . . . . . . . . . . . . . . . . . . . 301

11. Exponential Bounds on Resonances States. . . . . . . . . . . . . . . . 303

12. Width of Resonances. Proof of Theorem 6.3 . . . . . . . . . . . . . . . . . . . 305

13. Stability Estimate. . . . . . . . . . . . . . . . . . . . . . . 306

Appendix . . . . . . . . . . . . . . . . . . . . . . . . . . . . 312

References. . . . . . . . . . . . . . . . . . . . . . . 313

* Research support by NSERC under grant NA 7901 


\section{Introduction}

The purpose of article is to derive bounds on life-time of "unstable bound states" (=(quantum) resonances in modern terminology) of many-electron systems placed in constant electric fields. These bounds give a partial generalization of the classical Oppenheimer formula [Op, LL] obtained for the hydrogen atom. The latter formula was rigorously justified in [HaSi] who, after Oppenheimer, used separation of variables in parabolic coordinates and an ODE technique. This brings us to another task of this paper: to initiate a geometric theory of resonances in many-body systems. The main characteristic of resonance is its life-time $(=1 /$ width). In an important recent development the life-time of resonances in onebody systems was estimated in terms of length of certain minimal geodesics (between classical turning surfaces) in the Agmon Riemannian metric (see [BCD, CDKS, HS, HisSig 1, S 2]). (The latter is the Jacobi metric of an instanton and, consequently, the length of minimal geodesic is the classical action of the instanton.) The main point brought up in this paper is that the main contribution into the resonance life-time in the many-body case comes from an instanton-type action which depends on eigenvalues of subsystems and the geometry of manybody configurations. This interplay between the geometry of potentials, manybody geometry and many-body dynamics is the unique feature of quantum manybody systems.

Finally, we mention two features of the many-body Stark effect which make it an attractive model for studying the resonances: (a) There is a natural small parameter - the electric field strength (in contrast to quasiclassical theories) and (b) the corresponding perturbation theory is singular. Indeed, as the electric field is switched on, the spectrum (as well as other parameters) changes dramatically: the continuum fills the entire real axis, while the eigenvalues disappear. The fate of these eigenvalues and the mechanism of their turning into the resonances are the main objects of our discussion.

The results of this paper were announced and the proofs outlined in [S 4].

\section{Hamiltonian}

Consider an $N$ particle system in $\mathbb{R}^{v}$ with external interactions. Its Schrödinger operator is

$$
H=\sum_{i=1}^{N}\left(p_{i}^{2}+V_{i}\left(x_{i}\right)\right)+\Sigma V_{i j}\left(x_{i}-x_{j}\right) .
$$

It acts on $L^{2}\left(\mathbb{R}^{v N}\right)$. Here $x_{j} \in \mathbb{R}^{v}$ and $p_{j}=-i\left(\operatorname{grad}\right.$ in $\left.x_{j}\right)$. We interpret the $N$ moving particles as electrons and $V_{i}\left(x_{i}\right)$, as external potentials due to interactions with fixed nuclei. In the case of atoms and molecules in the approximation of infinitely heavy nuclei (in the latter case it is called the Born-Oppenheimer or adiabatic approximation), $V_{i}$ and $V_{i j}$ are Coulomb potentials (attractive and repulsive, respectively). We will not use a specific shape of these potentials but rather assume the following general properties:

(I) $V_{l}(y)$ are analytic in $Q \cap\left\{\zeta \in \mathbb{C}^{v} \mid\|\operatorname{Re} \zeta\|>R\right\}$ for $l=i$ and in $Q$ for $l=i j$ as $\Delta_{y}$-bounded operators. Here

$$
Q=\left\{\zeta \in \mathbb{C}^{\nu} \mid\|\operatorname{Im} \zeta\| \leqq \varepsilon\|\operatorname{Re} \zeta\|(1+\|\operatorname{Re} \zeta\|)^{-\eta}\right\}
$$


for some $\varepsilon>0$ and $1>\eta \geqq 0$ (see the figure below).

(II) $V_{l}(\zeta)$ and $y \cdot \nabla V_{i j}(\zeta)$ are $\Delta_{y}$-bounded with the relative bound 0 . Here $l$ is either $i$ or $i j$ and $y=\operatorname{Re} \zeta$. Besides $V_{i j}(y)$ are bounded for $y \neq 0$.

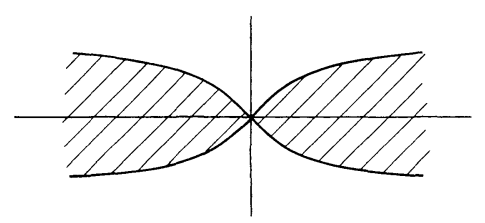

(III) $\left(\nabla^{n} V_{l}\right)(\zeta)=O\left(|\zeta|^{-\mu-n}\right)$ at $\infty$ for $n=0,1$ and some $\mu>0$, on the domain of analyticity of $V_{l}$. Here $l$ is either $i$ or $i j$.

(IV) $V_{i j}$ are repulsive along the direction $e$ of the field in the sense that

$$
\operatorname{Im} V_{i j}\left(z, y^{\perp}\right) \leqq 0 \quad \text { and } \quad \operatorname{Re} V_{i j}\left(z, y^{\perp}\right) \geqq 0 \quad \text { if } \quad \operatorname{Re} z \operatorname{Im} z \geqq 0,
$$

where $y$ is written as $\left(y \cdot e, y^{\perp}\right)$ with $y^{\perp}$, the projection of $y$ on $e^{\perp}$.

Under these conditions $H$ is self-adjoint on its natural domain and its spectrum looks like

$$
\operatorname{Spec}(H)
$$

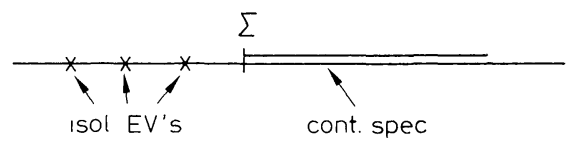

If our $N$-particle system is placed in a constant (homogeneous) electric field, whose strength is in the direction of a unit vector $e$ in $\mathbb{R}^{v}$, then its Hamiltonian becomes

$$
H_{f}=H-F \cdot x \text {. }
$$

Here $F=f\left(q_{1} e, \ldots, q_{N} e\right)$, where $f$ is the amplitude of the electric field strength and $-q_{i}<0$, the charge of the $i$-th particle. One can again show (see $[\mathrm{HeSi}]$ ) that $H_{f}$ is self-adjoint and its continuous spectrum fills the entire real axis (the latter result $I$ was able to locate only for one-body systems). Moreover, it has no bound states. For the hydrogen atom this was proven by Titchmarsh ([T], see $[\mathrm{AH}]$ for a oneparticle generalization) and for $N$-particle systems satisfying somewhat more general conditions than those of this paper, in [S 3].

\section{Instanton Metric}

In this section we define the Agmon-type Riemannian metric whose geodesics control the tunneling. The lengths of these geodesics can be interpreted as an instanton action. However, the corresponding instanton incorporates quantum data (ionization energies).

We begin with describing the geometry of many-particle configurations (see [Hu 2]). Let $B_{f}$ be the exterior of the sphere of radius $f^{-\beta}$ centered at the origin:

$$
B_{f}=\left\{x \in \mathbb{R}^{v N}|| x \mid>f^{-\beta}\right\} .
$$


It is convenient for us to pick $\beta>\left(1+\min \left(2, \frac{\mu}{2}\right)\right)^{-1}$, where $\mu$ is the rate of decay of potentials given in (III). Let $a=\{A, B\}$ be an ordered decomposition of $\{1, \ldots, N\}$ into subsets (called also subsystems or clusters). We define the domains

$$
\begin{gathered}
\Omega_{a, f}=\left\{x \in B_{f} \mid e \cdot x_{i}<n f^{-\beta} \forall i \in A,\right. \\
\left.e \cdot x_{j}>(n+1) f^{-\beta} \forall j \in B \text { for some } 1 \leqq n \leqq N\right\} .
\end{gathered}
$$

The collection of these sets together with the ball

$$
\left\{x \in \mathbb{R}^{v N}|| x \mid<2 f^{-\beta}\right\}
$$

forms an open covering on $\mathbb{R}^{v N}$. Indeed, for any $x \in \mathbb{R}^{v N}$ one of the $N+1$ intervals

$$
\left(-\infty, f^{-\beta}\right),\left(f^{-\beta}, 2 f^{-\beta}\right), \ldots,\left((N-1) f^{-\beta}, N f^{-\beta}\right),\left(N f^{-\beta}, \infty\right)
$$

contains no $e \cdot x_{i}$ 's. Let $A$ be the set of all indices $i$ such that $e \cdot x_{i}$ belong to one of the preceding intervals. Then, clearly $x \in \Omega_{a, f}$.

We introduce some notation. Let $H_{A, f}$ be the Hamiltonian of $A$ in the electric field and let $H_{A}=H_{A, 0}$. Let $E_{A}=\inf \sigma\left(H_{A}\right)$. We set

$$
V_{A, f}=\sum_{A} V_{i j}\left(x_{i}-x_{j}\right)-\sum_{A} F_{i} x_{i} \text {. }
$$

Due to the HVZ-theorem

$$
\Sigma=\min _{A \subset\{1, \ldots, N\}} E_{A}=\inf \sigma_{\mathrm{ess}}(H) .
$$

Assume our system is in the region $\Omega_{a, f}$. This means that it is broken into subsystems $A$ and $B$, with $A$, confined in the domain in which the electric field is either negligible or pulls it toward the origin. The subsystem $B$ is trying to escape into the region where the electric field pulls it toward $\infty$ overcoming the attraction by the nuclear potential. To give $B$ the maximal kinetic energy, $A$ is placed into the ground state. Thus the potential barrier for $B$ is

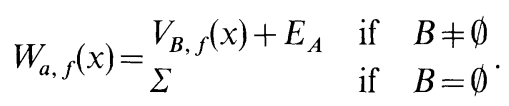

The $B=\emptyset$ case is introduced for a notational convenience. The instanton action for $B$ is given by the geodesic length in the instanton (imaginary time) Jacobi metric

$$
\left(W_{a, f}(x)-E\right)_{+} d x^{2},
$$

where $E$ is the total energy of the system. This is exactly the Agmon metric for the potential $W_{a, f}$. There is an ambiguity since the regions of overlap of different $\Omega_{a, f}$ are equipped now with several Riemannian structures. This is resolved by taking the smallest of the available metrics. Namely, introduce a partial order on the set of partitions:

$$
a \cong a^{\prime} \Leftrightarrow A \supseteqq A^{\prime}
$$

For each $x \in B_{f}$ we define the collection of partitions:

$$
A_{x, f}=\left\{a \mid x \in \Omega_{a, f}\right\} .
$$


Since $\Omega_{a, f}$ cover $B_{f}$, this set is not empty. $a \in A_{x, f}$ label different "gaps" in the linear system $e \cdot x_{1}, \ldots, e \cdot x_{N}$. Hence the set $A_{x, f}$ is completely ordered and therefore

$$
a(x) \equiv a_{f}(x) \equiv \min \left\{a \mid a \in A_{x, f}\right\}
$$

exists, and, of course, is unique. Now we can uniquely assign to each point the Riemannian metric reflecting the picture described above:

$$
d s^{2}=\left(W_{a(x), f}(x)-E\right)_{+} d x^{2} .
$$

Denote by $\varrho_{E, f}(x, y)$ the geodesic distance between $x$ and $y$ defined for this structure:

$$
\varrho_{E, f}(x, y)=\inf \{L(\gamma) \mid \gamma \cdot x \rightarrow y\},
$$

where the infimum is taken over all $L^{1}$-rectifiable curves from $x$ to $y$ (see e.g. [Ag, HisSig 2]) and

$$
L(\gamma)=\int_{0}^{1}\left(W_{a(\gamma), f}(\gamma)-E\right)_{+}^{1 / 2}\|\dot{\gamma}\| d s .
$$

The Riemannian metric above is discontinuous but bounded away from the coincidence planes for subsystems $B$. Besides, it is degenerate: it vanishes on the energetically allowed region. However, $\varrho_{E, f}$ is Lipschitz as can be easily shown using the triangle inequality and by replacing an intermediate geodesic by a straight path (cf. [Ag, HisSig 2]).

Let $\varrho_{E, f}(x)$ be the geodesic distance of $x$ to $O$ in the metric above.

Proposition 3.1. The geodesic distance $\varrho_{E, f}(x)$ is differentiable almost everywhere and

$$
\left|\nabla \varrho_{E, f}(x)\right| \leqq\left(W_{a(x), f}(x)-E\right)_{+}^{1 / 2}
$$

holds.

Proof. By a standard argument (see e.g. [Ag, HisSig 2]), $\varrho_{E, f}$ is Lipschitz and obeys

$$
\lim _{h \rightarrow 0} \frac{1}{\|h\|}\left|\varrho_{E, f}(x+h)-\varrho_{E, f}(x)\right| \leqq\left(W_{a(x), f}(x)-E\right)_{+}^{1 / 2} .
$$

By Rademacher's theorem (see e.g. [Mo]) $\varrho_{E, f}$ is then differentiable almost everywhere and by a result of $[\mathrm{Ag}]$ it obeys (3.11).

\section{Energetically Forbidden Region}

Let $E<\Sigma$. Denote by $K_{E, f}$ the support of the instanton metric (3.8):

$$
K_{E, f}=\left\{x \in \mathbb{R}^{v N} \mid W_{a(x), f}>E\right\} .
$$

We show in Sect. 7 that the "energy conservation law" breaks down on $K_{E, f}$. Consequently, we call $K_{E, f}$ the energy forbidden region. In this section we study its geometry. Let $\hat{F}=F /\|F\|$ and introduce

$$
\Pi_{\gamma}=\left\{x \in B_{f} \mid \hat{F} \cdot x \leqq \gamma^{-1}\right\} .
$$


We have the following embeddings:

$$
\begin{gathered}
\Pi_{\alpha f} \subset K_{E, f} \quad \text { for } \alpha \text { suffic. large, } \\
K_{E, f} \cap \Omega_{\delta} \subset \Pi_{\beta f} \quad \text { for } \beta \text { suffic. small. }
\end{gathered}
$$

Here

$$
\Omega_{\delta}=\left\{x \in \mathbb{R}^{v N}\left|\min _{B}\right| x_{i}-x_{j} \mid>\delta\right\} .
$$

Here $\beta$ depends on $\delta: \beta \rightarrow 0$ as $\delta \rightarrow 0$. To prove (4.3) we observe that $\Pi_{\alpha f}$ is covered by $\Omega_{a, f} \cap \Pi_{\alpha f}$ with $B \neq \emptyset$. Since

$$
V_{B, f} \geqq V_{B}-\text { const } / \alpha \geqq- \text { const } / \alpha \quad \text { on } \quad \Omega_{a, f} \cap \Pi_{\alpha f},
$$

where $V_{B}=V_{B, 0}$, and since $E_{A}>\mathrm{E}$, we have that

$$
W_{a, f}(x)>E \quad \text { on } \quad \Omega_{a, f} \cap \Pi_{\alpha f} \text { for } \alpha \text { suffic. large. }
$$

Hence (4.3) follows. To prove (4.4) let $x \in K_{E, f} \cap \Omega_{\delta}$. Then there is a such that $x \in \Omega_{a, f}$ and $W_{a, f}>E$. Next, note that

$$
V_{B}(x) \leqq \text { const if } \min _{B}\left|x_{i}-x_{j}\right|>\delta .
$$

This together with the previous inequality yields that

$$
\hat{F} \cdot x_{B} \leqq \text { const } / f \text {. }
$$

Here $\left(x_{B}\right)_{i}=x_{i}$ if $i \in B$ and $=0$ if $i \notin B$. Moreover, since $x \in \Omega_{a, f}$, we have that

$$
e \cdot x_{i}<\text { const } / f^{\beta} \text { for all } i \in A \text {. }
$$

Hence, if $x \in$ left-hand side of (4.4), we have

which implies (4.4).

$$
\hat{F} \cdot x \leqq \text { const } / f \text {, }
$$

Relations (4.3) and (4.4) show that $K_{E, f}$ separates the bounded interior region $B_{f}^{c}$ from an unbounded exterior region (lying outside of $\Pi_{\alpha f}$ with $\alpha$ sufficiently large). To get a feeling about the shape of $K_{E, f}$ we consider one of its components

$$
\left\{x \in B_{f} \mid a(x)=a\right\} \cap K_{E, f} .
$$

Its exterior boundary is defined by the equation

$$
F \cdot x_{B}=V_{B}\left(x^{B}\right)-E_{A}+E,
$$

where $x^{B}$ stands for the set of interior coordinates for the subsystem $B$. Observe that $\hat{F} \cdot x_{B}$ is the smallest when the particles in $B$ are far apart from each other and it is the largest, namely $\infty$, if some of the particles in $B$ coincide. As a result we have the following picture (not to scale!).

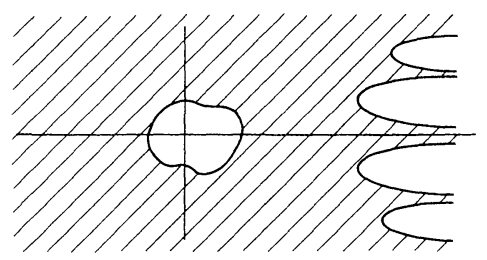


We denote by $S_{E, f}^{+}$the exterior boundary of $K_{E, f}$, i.e. the part of the boundary of $K_{E, f}$ lying outside $\Pi_{\alpha f}$ for $\alpha$ sufficiently large. Denote by $\sigma_{E, f}$ the length of minimal geodesic from the origin to $S_{E, f}^{+}$. A simple estimate based on (4.3) shows that $\sigma_{E, f}=O\left(f^{-1}\right)$.

Finally we show that

$$
\operatorname{dist}\left(x, S_{E_{0}, f}^{+}\right)=O\left(\left|E-E_{0}\right| f^{-1}\right) \forall x \in S_{E, f}^{+} .
$$

To demonstrate this estimate we note first that

$$
x \in S_{E, f}^{+} \Rightarrow x \in \Omega_{a, f} \quad \text { and } \quad W_{a, f}(x)=E
$$

for some $a$. Consider a point $x_{0}$ in the $a$-component of $S_{E_{0}, f}^{+}$with the same $x^{B}$-coordinates and with the same $F^{\perp}$-projection of $x_{B}$. In other words, we change the $\hat{F} \cdot x_{B}$-coordinate of $x$ and keep the other coordinates fixed till the new point $x_{0}$ obeys

$$
x_{0} \in \Omega_{a, f} \quad \text { and } \quad W_{a, f}\left(x_{0}\right)=E_{0} .
$$

Thus $x_{0} \in S_{E_{0}, f}^{+}$. By the construction

$$
W_{a, f}(x)-W_{a, f}\left(x_{0}\right)=F \cdot\left(x_{B}-x_{0, B}\right)=\|F\|\left\|x-x_{0}\right\| .
$$

On the other hand

$$
E-E_{0}=W_{a, f}(x)-W_{a, f}\left(x_{0}\right) .
$$

The last two relations give

$$
\left\|x-x_{0}\right\|=O\left(\left|E-E_{0}\right| f^{-1}\right),
$$

which proves (4.9).

\section{Spectral Deformations}

A rigorous theory of resonances was started in [AC, BC, Sim 1] for dilationanalytic potentials. A more general theory has been developed in [Sim 2, Sig 1, Hun 1, Cyc, HisSig 1, BCD]. In this section we outline a general framework for the definition of resonances (for more details see the lecture notes [HisSig 2]).

Let $v$ be a smooth vector field in $\mathbb{R}^{v N}$ obeying sup $\|D v(x)\|<\infty$. Let $g_{\theta}$ be the global flow generated by $v$ or its first approximation, $x+\theta v(x)$, i.e. the shift by $v$. Define the one-parameter family of unitary operators

$$
U(\theta): \psi \rightarrow \sqrt{\mathrm{Jac}} \psi \circ g_{\theta},
$$

where Jac is the Jacobian of the map $x \rightarrow g_{\theta}(x)$. If $g_{\theta}$ is the flow, then $U(\theta)$ is a group:

$$
U(\theta+\eta)=U(\theta) U(\eta) .
$$

Note that $U(\theta)$ leaves $D(\Delta)$ invariant.

Given a Schrödinger operator $H=-\Delta+V(x)$ we associate with it the oneparameter family of self-adjoint operators

$$
H(\theta)=U(\theta) H U(\theta)^{-1}
$$


[conjugation of $H$ by $U(\theta)$ ], defined on the same domain $D(H)$. We call $H(\theta)$ a deformation of $\mathbf{H}$ by the vector field $v$. It is easy to write $H(\theta)$ out explicitly

$$
H(\theta)=\left\|p_{\theta}\right\|^{2}+V \circ g_{\theta},
$$

where

$$
p_{\theta}=\left(\frac{\partial g_{\theta}}{\partial x}\right)^{-1 T} p+\frac{i}{2} \nabla\left(\operatorname{lndet} \frac{\partial g_{\theta}}{\partial x}\right) .
$$

Of course, the operators $H(\theta), \theta \in \mathbb{R}$, and $H$ are entirely equivalent, they have the same spectra, multiplicities, etc. However, this changes dramatically if $\theta$ moves into the complex plane. The latter is the case of interest for us and to tackle it we impose the following restrictions on $H(\theta)$ :

(A) $H(\theta), \theta \in \mathbb{R}$, is a restriction of an analytic of type $A$ family, $H(\theta)$, defined on a neighbourhood, $A$, of $\theta=0$.

(B) There is an open connected set, $\Omega \subset \mathbb{C}$, having non-empty intersections with $\mathbb{C}^{+}$and $\mathbb{C}^{-}$and such that

$$
\begin{aligned}
& \Omega \cap \mathbb{C}^{+} \cap \sigma_{\text {ess }}(H(\theta))=\emptyset \text { for all } \theta \in A \cap \overline{\mathbb{C}^{+}}, \\
& \Omega \cap \sigma_{\text {ess }}\left(H\left(\theta_{0}\right)\right)=\emptyset \text { for some } \theta_{0} \in A \cap \mathbb{C}^{+} .
\end{aligned}
$$

Under these conditions, the discrete spectrum of $H(\theta)$ with $\theta \in A \cap \mathbb{C}^{+}$has the following properties:

(i) The discrete spectrum inside of $\Omega$ is located in $\Omega \cap \overline{\mathbb{C}^{-}}$.

(ii) $\sigma_{d}(H(\theta)) \cap \mathbb{R} \cap \Omega=\sigma_{p p}(H) \cap \Omega$.

(iii) The discrete spectrum inside of $\Omega$ is independent of $\theta_{0}$ and of the vector field $v$ used, provided different analytic sets $A_{U}$ have a dense intersection.

Here $A_{U}$ is the set of vectors analytic with respect to the family $U(\theta)$. Thus, the complex eigenvalues of $H(\theta), \operatorname{Im} \theta>0$, inside of $\Omega$ lie in $\mathbb{C}^{-}$and are intrinsic to $H$. They are closely related to the embedded eigenvalues of $H$ : under perturbation, complex eigenvalues of $H(\theta)$ might become embedded eigenvalues of $H$ and vice versa. The complex eigenvalues of $H(\theta)$ with $\operatorname{Im} \theta>0$ (inside of $\Omega$ ) are called the (spectral) resonances of $H$.

Now we define some concrete deformations $H_{f}(\theta)$ of the Stark Hamiltonian $H_{f}$. Following the general procedure outlined above, the Stark resonances are defined as complex eigenvalues of $H_{f}(\theta)$ with $\operatorname{Im} \theta>0$.

Let $v_{i}=w\left(x_{i} \cdot e\right)$, where $w$ is a smooth bounded function obeying:

$$
\begin{aligned}
& \left|w^{(n)}\right| \leqq \text { const } f^{\beta n-\gamma} \quad \text { for } \quad n=1,2,3 \text {, } \\
& w(s)=\begin{array}{ccc}
0 & \text { for } & s \leqq \frac{1}{2 f^{\beta}} \\
f^{-\gamma} & \text { for } & s \geqq \frac{1}{f^{\beta}},
\end{array}
\end{aligned}
$$

with $\gamma>1-\beta$. Let $E_{0}$ be an eigenvalue of $H$ under consideration. Denote by $S_{a f}$ the geodesic sphere of radius $\sigma_{E_{0}, f}-(a f)^{-\beta}$, centered at the origin. Here we use instead of original metric (3.8), the metric defined with $V_{i j}(y)$ smoothed out inside of a sufficiently small ball around $y=0$. This removes inessential difficulties 
caused by possible singularities of $V_{i j}$. The following property of $S_{f}$ will play an important role later:

$$
S_{f} \text { lies inside of } S_{E, f}^{+} \text {and } \operatorname{dist}\left(S_{f}, S_{E, f}^{+}\right)=O\left(f^{-\beta}\right),
$$

provided $E-E_{0}=o\left(f^{1-\beta}\right)$. This property follows from (4.9). Let $\chi_{f}$ be a smooth cut-off function, $0 \leqq \chi_{f} \leqq 1$ which is chosen so that

$$
\chi_{f}=\begin{array}{ll}
0 & \text { inside } S_{f} \\
1 & \text { outside } S_{2 f}
\end{array},
$$

and moreover it satisfies

$$
\nabla^{n} \chi_{f}=O\left(f^{n \beta}\right)
$$

Define the vector field

$$
v=\left(v_{1} e, \ldots, v_{N} e\right) \chi_{f},
$$

Denote by $H_{f}(\theta)$ the deformation of $H_{f}$ by vector field (5.9) through the shift $g_{\theta}=\mathrm{id}+\theta v$. A simple computation based on (5.2) shows that $H_{f}(\theta)$ [see Eqs. (5.10)-(5.13)] is of the form

$$
\begin{aligned}
H_{f}(\theta)= & H_{f}+2^{\text {nd }} \text { order diff. opr. with comp. supported coeff. } \\
& + \text { bounded multipl. opr. }
\end{aligned}
$$

Thus a result of [S 3] shows that $H_{f}(\theta)$ have the same domain as $H_{f}$. Moreover, an examination of the expression for $H_{f}(\theta)$ reveals that $H_{f}(\theta) u$ is analytic in $\theta$ in a neighbourhood of $\theta=0$ for any $u \in D\left(H_{f}\right)$. Hence $H_{f}(\theta)$ is an analytic family in a vicinity of $\theta=0$ of type $A$ in the sense of Kato with the same domain as $H_{f}$. We will use the notation:

$$
H(f, \phi)=H_{f}(i \phi)
$$

A simple computation gives

$$
H(f, \phi)=T_{\phi}+\Sigma V_{i, f}(\phi)+\Sigma V_{i j}(\phi),
$$

where

$$
T_{\phi}=\left\|p_{i \phi}\right\|^{2}
$$

with $p_{\theta}$ defined by (5.3), (5.9), with $g_{\theta}=\mathrm{id}+\theta v$ and

$$
V_{j, f}(\phi)=V_{j, f}\left(x_{j}+i \phi v_{j} \chi_{f} e\right), \text { etc. }
$$

We show that the spectrum of $H(f, \phi)$ with $\phi>0$ looks like

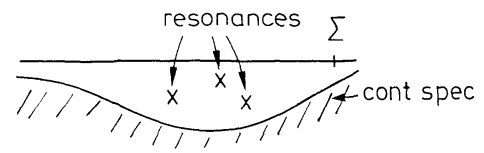

This picture holds without the cut-off function $\chi_{f}$ as well. The role of the latter is to keep the deformed Hamiltonian real in the region in which we estimate the tunneling. 


\section{Main Result}

Analytically the most difficult part of our analysis is an estimate on the resolvent of $H(f, \phi)$ in a neighbourhood of isolated eigenvalues of $H$. We call this estimate the stability estimate since it plays the key role in the proof of stability of eigenvalues of $H(f, \phi)$, i.e. the existence of the resonances and bound states of $H_{f}$. This type of estimates (and under similar names) was isolated in [K, $\mathrm{VH}]$ (see [Hu 2] for new developments). To formulate this estimate we need some definitions and notations. We consider the annulus

$$
\frac{1}{2} f^{v} \leqq\left|z-E_{0}\right| \leqq f^{v}
$$

in the energy plane, around a given isolated eigenvalue, $E_{0}$, of the unperturbed Hamiltonian $H$. Here $v$ obeys

$$
1-\gamma<v<\beta
$$

Let $E^{\prime}=E_{0}+f^{\alpha}$ with

$$
1-\beta<\alpha<\varepsilon,
$$

where

$$
\varepsilon=\min (2 \beta, \mu \beta, \beta-\gamma,(1+\mu) \beta-1) .
$$

For a smooth function $\xi$ we define the norm

$$
\|u\|_{1, \xi}=\|u\|+\|p \xi u\| \text {. }
$$

In this section we assume the potentials obey the conditions (I)-(IV). We formulate now the stability estimate theorem.

Theorem 6.1. Let $\xi,|\xi| \leqq 1$ be supported inside $S_{E^{\prime}, f}^{+}$. Then for any $u \in C_{0}^{\infty}$,

$$
\|(H(f, \phi)-z) u\| \geqq \delta f^{v}\|u\|_{1, \xi}
$$

for $z$ in annulus (6.1), $f$ sufficiently small and $\delta>0$, independent of $z, f$ and $u$.

The proof of this theorem is given in Sect. 13.

Now we turn to an existence result which is a by-product of our estimates.

Theorem 6.2. Near each isolated eigenvalue $E_{0}$ of $H$ there are eigenvalues and resonances $E_{i}(f)$ of $H_{f}$ so that

$$
E_{i}(f) \rightarrow E_{0} \quad \text { as } \quad f \rightarrow 0 .
$$

Moreover, the total multiplicity of $E_{i}(f)$ is equal to that of $E_{0}$.

This theorem extends to the class of potentials (I)-(IV) the result of [HeSi] (see also [ $\mathrm{Hu} 2]$ for a different, geometric proof). Note that for this class of potentials a result of [S 3] says that $H_{f}$ has no eigenvalues. Thus the combination of both results implies the existence of resonances for $H_{f}$. The proof of this theorem is given in Sect. 9.

Our main result is the following. 
Theorem 6.3. Let $E_{i}(f)$ be the resonances of $H_{f}$ splitting from a given eigenvalue $E_{0}$ of $H_{f}$ (see Theorem 6.2). Then their widths are bounded as

$$
\left|\operatorname{Im} E_{i}(f)\right| \leqq C f^{2 \beta-\alpha} \exp \left[-2 \sigma_{E_{0}, f}+O\left(f^{-\beta}\right)\right]
$$

for all $f$ sufficiently small and $\alpha<\min (\mu \beta, \beta-\gamma)$.

The proof of this theorem is given in Sects. 7-13. The key ingredient in estimating the resonances $E_{i}(f)$ is an exponential bound on the resonance states, i.e. eigenfunctions of $H(f, \phi)$. To derive the latter we compare $H(f, \phi)$ in a region of interest with an auxiliary self-adjoint operator $H_{f}^{0}$. In our approach we use an Agmon-type method to derive first exponential bounds on eigenfunctions of $H_{f}^{0}$ and then use a perturbative argument to compare two sets of eigenfunctions and to obtain exponential bounds on the resonance states.

\section{Energy Inequalities}

In this section we study the support $K_{E, f}$ of the Stark instanton metric (3.8). First we derive another representation for it and then we discuss its interpretation. We begin with inequalities for the potential barriers.

Lemma 7.1. For $x \in \Omega_{a, f} \cap \Omega_{a^{\prime}, f}$ and $f$ sufficiently small,

$$
W_{a, f}(x) \geqq W_{a^{\prime}, f}(x) \quad \text { if } a \supset a^{\prime} .
$$

Proof. Let $B^{\prime} \neq \emptyset$. The case $B^{\prime}=\emptyset$ is simpler. Recall that the ordering $a \supset a^{\prime}$ means that $A \subset A^{\prime}$ and $B \supset B^{\prime}$. Hence

$$
E_{A}>E_{A^{\prime}} \text { and } \quad V_{B}(x) \geqq V_{B^{\prime}}(x) .
$$

Next, since $B \backslash B^{\prime} \subset A^{\prime}$, the particles in $B \backslash B^{\prime}$ are at the distance $O\left(f^{-\beta}\right)$ from the origin. Hence

$$
F \cdot x_{B}=F \cdot x_{B^{\prime}}+O\left(f^{1-\beta}\right) .
$$

Thus (7.1) holds for $f$ sufficiently small.

Lemma 7.2 (energy inequalities). For $f$ sufficiently small

$$
H_{f} \geqq W_{a, f}(x)+O\left(f^{1-\beta}\right) \quad \text { on } \Omega_{a, f} .
$$

The inequality is understood in the sense of quadratic forms on $C_{0}^{\infty}\left(\Omega_{a, f}\right)$.

Proof. Let first $B=\emptyset$, i.e. we consider the left half of $B_{f}$ :

$$
\left\{x \in B_{f} \mid \widehat{F} \cdot x \leqq N f^{-\beta}\right\} .
$$

Since in this domain the electric potential, $-F \cdot x$, is either $O\left(f^{1-\beta}\right)$, and therefore negligible, or positive, the $N$-body geometric analysis (see e.g. [CFKS]) yields

$$
H_{f} \geqq \Sigma-O\left(f^{\beta \mu}\right) \quad \text { on }(7.5) .
$$

Now we consider the other half of $B_{f}$ :

$$
\left\{x \in B_{f} \mid \hat{F} \cdot x \geqq N f^{-\beta}\right\}=\left\{x \in \mathbb{R}^{v N} \mid \hat{F} \cdot x \geqq N f^{-\beta}\right\} .
$$


It is covered by $\Omega_{a, f}$ with $B \neq \emptyset$. Consider cluster decompositions of the Hamiltonian:

$$
H_{f}=H_{A, f} \otimes 1+1 \otimes H_{B, f}+I_{a},
$$

where $a$ is the cluster decomposition $\{A, B\}$ and $I_{a}$ is the intercluster interaction:

$$
I_{a}=\sum_{i \in A, j \in B} V_{i j}\left(x_{i}-x_{j}\right) .
$$

Using that on $\Omega_{a, f}$ (in the sense of quadratic forms)

$$
\begin{gathered}
H_{A, f} \geqq H_{A}+O\left(f^{1-\beta}\right), \\
H_{B, f} \geqq V_{B, f}+O\left(f^{\beta \mu}\right)
\end{gathered}
$$

and $H_{A} \geqq E_{A}$ and

$$
I_{a}=O\left(f^{\beta \mu}\right),
$$

and using that $\min (1-\beta, \mu \beta)=1-\beta$ (for $\beta$ defined in Sect. 3), we arrive at (7.4).

If $H_{f}$ is localized around $E$, we obtain the energy inequality

$$
E \geqq W_{a, f}+O\left(f^{1-\beta}\right) \quad \text { on } \Omega_{a, f} .
$$

This shows that on $K_{E, f}$ the energy conservation law breaks down.

Corollary 7.3 (combined energy inequality).

$$
H_{f} \geqq W_{a(x), f}(x)-C f^{1-\beta} \quad \text { on } B_{f} .
$$

Proof. Let $\left\{j_{a, f}\right\}$ be a partition of unity associated with the covering $\left\{\Omega_{a, f}\right\}$ :

$$
\sum\left(j_{a, f}\right)^{2}=1 \text { on } B_{f}
$$

and

$$
\operatorname{suppj}_{a, f} \subset \Omega_{a, f} \text {. }
$$

Besides, since the scale of $\Omega_{a, f}$ is $f^{-\beta}$ we can arrange that

$$
\nabla^{n} j_{a, f}=O\left(f^{n \beta}\right) .
$$

Applying the IMS localization formula (see e.g. [CFKS]) with this partition yields

$$
H_{f}=\sum j_{a, f} H_{f} j_{a, f}-\sum\left|\nabla j_{a, f}\right|^{2}
$$

Applying Lemma 7.2 to each term in the sum and using estimates (7.1) and (7.17) and the relation

$$
\min (1-\beta, \mu \beta, 2 \beta)=1-\beta
$$

(for $\beta$ defined in Sect. 3), we arrive at (7.14).

\section{Hamiltonian $\boldsymbol{H}_{f}^{0}$}

In this section we construct auxiliary Hamiltonians $H_{f}^{i}$ with which we compare the original Hamiltonian $H(f, \phi)$. The operator $H_{f}^{0}$ is self-adjoint. It agrees $H(f, \phi)$ on the interior region and on the energetically forbidden region excluding a small 
neighbourhood of the exterior boundary of the latter. Hence the contribution of tunneling in both cases is almost the same. The Hamiltonian $H^{\prime}(f, \phi)$ agrees with $H(f, \phi)$ in the exterior of $K_{E, f}$ and is used to control the behaviour of the latter operator there.

We begin with introducing the cut-off functions. Let $\chi_{f}^{(i)}$ be smooth functions such that $0 \leqq \chi_{f}^{(i)} \leqq 1$ and

$$
\chi_{f}^{(0)}=0 \text { outside } S_{f} \text { and }=1 \text { inside } S_{f / 2},
$$

and

$$
\chi_{f}^{(1)}=0 \text { outside } B_{f} \text { and }=1 \text { in } B_{f / 2} .
$$

We introduce now the new total potentials

$$
V_{f}^{(0)}=V-F \cdot x \chi_{f}^{(0)},
$$

and

$$
V_{f}^{(1)}=V_{f} \chi_{f}^{(1)}
$$

and the corresponding Hamiltonians

$$
H_{f}^{i}=p^{2}+V_{f}^{(i)} .
$$

We denote by $H^{\prime}(f, \phi)$ the deformation of $H_{f}^{\prime}$ by the vector field (5.9). Hence

$$
H^{\prime}(f, \phi)=T_{\phi}+V(f, \phi) \chi_{f}^{(1)},
$$

where $V(f, \phi)$ is the total deformed potential.

Proposition 8.1. The spectrum of $H_{f}^{0}$ in the disc $\left|z-E_{0}\right|<f^{v}$ consists of discrete eigenvalues $\mu_{i}(f)$ obeying

$$
\mu_{i}(f)-E_{0}=O\left(f^{1-\varepsilon}\right)
$$

for any $\varepsilon>0$. Moreover, the total multiplicity of the $\mu_{i}$ 's is equal to that of $E_{0}$.

Proof. First we study the continuous spectrum. Let $W_{a, f}^{0}$ be defined in the same way as $W_{a, f}$ but with $F \cdot x$ replaced by $F \cdot x \chi_{f}^{(0)}$.

Lemma 8.2. The following inequality holds on $B_{f}$ in the sense of quadratic forms

$$
H_{f}^{0} \geqq E^{\prime}-C f^{1-\beta},
$$

provided $f$ is sufficiently small.

Proof. We have exactly as in Corollary 7.3,

$$
H_{f}^{0} \geqq W_{a(x), f}^{0}(x)-C f^{1-\beta}
$$

on $B_{f}$ (in the sense of quadratic forms). We have by the definition of $\chi_{f}^{0}$,

$$
W_{a(x), f}^{0}(x) \geqq E^{\prime} \quad \text { on } \operatorname{supp} \chi_{f}^{0} .
$$

On the other hand

$$
\begin{aligned}
W_{a(x), f}^{0}(x) & =W_{a(x), 0}^{0}(x) \quad \text { on } \quad \mathbb{R}^{\nu N} \backslash \operatorname{supp} \chi_{f}^{0} \\
& \geqq \Sigma .
\end{aligned}
$$


Thus, since $\Sigma>E^{\prime}$.

$$
W_{a(x), f}^{0}(x) \geqq E^{\prime} .
$$

Estimates (8.9) and (8.12) yield (8.8).

Thus by the Weyl criterion and local compactness (see e.g. [CFKS, HisSig 2]) $H_{f}^{0}$ has no continuous spectrum in $\left(-\infty, E^{\prime}-C f^{1-\beta}\right)$. Now the proof of the proposition follows [Sim 3, HisSig 1]: it uses the min-max principle and the exponential bounds on eigenfunctions of $H_{f}^{0}$ proven in Sect. 10 and the known DHSV bounds on the eigenfunctions of $H$ (see [CFKS]).

\section{Stability of Resonances}

In this section we prove a stability result for eigenvalues of $H(f, \phi)$. It implies, in particular the existence of resonances or/and bound states of $H_{f}$ in a vicinity of discrete eigenvalues of $H$. Recall, that a result of [S 3] rules out the latter possibility (i.e. the bound states).

The stability estimate (Theorem 6.1) shows that annulus (6.1) is in the resolvent sets of $H(f, \phi)$. Proposition 8.1 shows the same for $H_{f}^{0}$. Let $\Gamma$ be a contour around $E_{0}$ lying in this annulus. Define the Riesz projections

$$
P(f, \phi)=\frac{1}{2 \pi i} \oint_{\Gamma}(z-H(f, \phi))^{-1} d z
$$

and

$$
P_{0}(f)=\frac{1}{2 \pi i} \oint_{\Gamma}\left(z-H_{f}^{0}\right)^{-1} d z
$$

They project onto the eigenspaces corresponding to the eigenvalues in the disc $\left|z-E_{0}\right|<\frac{1}{2} f^{v}$. The main result of this section is the following

\section{Theorem 9.1.}

$$
\left\|P(f, \phi)-P_{0}(f)\right\| \rightarrow 0 \quad \text { as } \quad f \rightarrow 0 .
$$

Proof. First, we introduce a smooth partition of unity $\left\{\phi_{i}\right\}$ normalized as

$$
\Sigma \phi_{i}=1
$$

and localized as follows:

$$
\begin{aligned}
\phi_{0} & =0 \text { outside } S_{f / 2} \\
& =1 \text { inside } S_{f / 4} .
\end{aligned}
$$

The localization of $\phi_{1}$ is corresponding. We also require that

$$
\nabla^{n} \phi_{i}=O\left(f^{n \beta}\right) \text {. }
$$

By the definition

$$
V_{f}^{(i)}=V_{i} \quad \text { on } \operatorname{supp} \phi_{i}
$$


In what follows we omit the arguments $f$ and $\phi$. Let $R$ and $R_{i}$ be the resolvents of $H$ and $H^{i}$, respectively. We use the geometric resolvent equation (see [CFKS, S5]):

$$
R+\Sigma R_{i} W_{i} R=\Sigma \phi_{i} R_{i},
$$

where, due to (9.7),

$$
W_{i}=\left[p^{2}, \phi_{i}\right]=2 p \cdot \nabla \phi_{i}-\Delta \phi_{i} .
$$

Using this relation, estimate (9.6) and the stability estimate (6.4) with $\xi=f^{-\beta} \nabla \phi_{i}$, we arrive at

$$
\left\|R_{i} W_{i} R\right\| \leqq C f^{\beta-v}\left\|R_{i}\right\| .
$$

A simplified version of the proof of Theorem 6.1 produces the following result:

$$
\left\|R_{1}(z)\right\| \leqq C f^{-v} \text { for }\left|z-E_{0}\right|<f^{v} .
$$

Moreover, the spectral theorem implies

$$
\left\|R_{0}(z)\right\| \leqq C f^{-v} \text { for } z \text { in (6.1). }
$$

The last two estimates together with (9.8) and (9.10) yield

$$
\left\|R-\Sigma \phi_{i} R_{i}\right\| \leqq C f^{\beta-2 v}
$$

for $z$ in (6.1). Moreover, due to (9.11), we have

$$
\oint_{\Gamma} R_{1} d z=0 .
$$

Thus integrating Eq. (9.8) over $\Gamma$, using (9.13) and (9.14) and using that $|\Gamma|=O\left(f^{v}\right)$ we arrive at

$$
\left\|P-\phi_{0} P_{0}\right\| \rightarrow 0 .
$$

Finally, due to the exponential bounds proven in Sect. 10,

$$
\left(1-\phi_{0}\right) P_{0} \rightarrow 0 \text { as } f \rightarrow 0 .
$$

The last two relations imply (9.3).

Theorems 8.1 and 9.1 imply Theorem 6.2.

\section{Exponential Bounds on Eigenfunctions of $\boldsymbol{H}_{\boldsymbol{f}}^{\mathbf{0}}$}

In this section we derive exponential bounds on the eigenfunctions $\phi_{i, f}$ of $H_{f}^{0}$ corresponding to the isolated eigenvalues $\mu_{i}(f)$ (splitting from $\left.E_{0}\right)$. This is the first step in derivation of exponential bounds on the resonance states.

Recall, $\varrho_{E, f}$ is the geodesic distance from $x$ to the origin [in the Riemannian metric (3.8)]. From now on we use notation $\langle A\rangle_{u}=\langle u, A u\rangle$. Whenever it does not cause a confusion we omit the subindex $u$ on the left-hand side.

Theorem 10.1. The eigenfunctions $\phi_{i, f}$ of $H_{f}^{0}$ corresponding to the eigenvalues splitting from $E_{0}$ verify the estimate

$$
\int e^{2 \varrho_{E_{0}, f}+O\left(f^{\alpha-1}\right)}\left|V^{n} \phi_{i, f}\right|^{2} \leqq \text { const } f^{2 \beta-\alpha},
$$

provided $f$ is sufficiently small, $n=0,1$. 
Proof. The method we use is, essentially due to Agmon [Ag] (see also [Sim 3]). The key point of the Agmon method is a positivity estimate. In our case this is

$$
\left\langle H_{f}^{0}-|\nabla \eta|^{2}-\mu_{i}(f)\right\rangle_{u} \geqq b f^{\alpha}\|u\|_{1}^{2},
$$

for some $b>0$ (independent of $f$ ), provided $u$ is supported in $B_{f}$ and, with $E^{\prime}=E_{0}+f^{\alpha}$,

$$
|\nabla \eta(x)|^{2} \leqq\left(W_{a(x), f}(x)-E^{\prime}\right)_{+} .
$$

Since $E_{A}=\inf H_{A}$ we have for any $\lambda>0$,

$$
H_{A} \geqq E_{A}+\lambda\left(H_{A}-E_{A}\right) \text {. }
$$

Since $p_{A}^{2}$ is $H_{A}$-bounded there is $c>0$ such that

$$
H_{A} \geqq E_{A}+\lambda\left(p_{A}^{2}-c\right)
$$

for any $\lambda>0$. Replacing in the proof of Corollary 7.3 the inequality $H_{A} \geqq E_{A}$ by (10.5) and retaining $p_{B}^{2}$ in the $H_{B, f}$-term and then adopting the resulting proof to the operator $H_{f}^{0}$, we obtain the following improvement of inequality (8.9): there is $c>0$ such that for any $\lambda>0$,

$$
H_{f}^{0} \geqq W_{a(x), f}^{0}(x)+\lambda p^{2}-\lambda c-C f^{1-\beta} .
$$

Note that due to (5.6) and $\beta>1-\alpha, S_{f}$ lies inside of $S_{E^{\prime}, f}^{+}$. Taking into account the definition of $\chi_{f}^{(0)}$ and considering separately the interior of $S_{E^{\prime} . f}^{+}$and the exterior of $S_{E^{\prime}, f}^{+}$, we obtain

$$
W_{a(x), f}^{0} \geqq E^{\prime}+\left(W_{a(x), f}-E^{\prime}\right)_{+} .
$$

The last three inequalities (with $\lambda=\frac{1}{4 c} f^{\alpha}$ ) combined with (8.7) produce, for $f$ sufficiently small, (10.2).

Now we show how positivity estimate (10.2)-(10.3) leads to exponential bound (10.1). We drop the argument and subindex $f$ for the rest of this proof. Let $j$ be a smooth non-negative function equal to 0 on $B_{2 f}^{c}$ and equal to 1 on $B_{f / 2}^{c}$. Our task is to prove that

$$
\left|\left\langle H^{0}-|\nabla \eta|^{2}-\mu_{i}\right\rangle_{u}\right| \leqq 2 \sup |\nabla j|^{2}\left\|\phi_{i}\right\|^{2},
$$

where $u=j e^{\eta} \phi_{i}$. Here $\eta$ is assumed to be a smooth bounded function vanishing on $B_{f}$. Note that $\phi_{\eta} \equiv e^{\eta} \phi_{i} \in D\left(H^{0}\right)$. We define the gauged Hamiltonian

$$
H_{\eta}=e^{\eta} H^{0} e^{-\eta} \text {. }
$$

It can be written as

$$
H_{\eta}=H^{0}-|\nabla \eta|^{2}+i(\nabla \eta \cdot p+p \cdot \nabla \eta) .
$$

Since $\phi_{i}$ is an eigenfunction of $H^{0}$ we have that $\phi_{\eta}$ is an eigenfunction of $H_{\eta}$ :

$$
\left(H^{\eta}-\mu_{i}\right) \phi_{\eta}=0 \text {. }
$$

Commuting $H_{\eta}-\mu_{i}$ through $j$ and using this equation, we obtain

$$
\left\langle H_{\eta}-\mu_{i}\right\rangle_{u}=\left\langle j\left[H_{\eta}, j\right]\right\rangle_{\phi_{\eta}} .
$$


Using representation (10.10) of $H_{\eta}$, we compute

$$
\operatorname{Re}\left\langle j\left[H_{\eta}, j\right]\right\rangle=\left\langle\left[j,\left[p^{2}, j\right]\right]+j \nabla \eta \cdot \nabla j\right\rangle .
$$

Computing the commutator on the right-hand side and using that

$$
\eta=0 \quad \text { on } \operatorname{supp}(\nabla j)
$$

we obtain

$$
\operatorname{Re}\left\langle H_{\eta}-\mu_{i}\right\rangle_{u}=\left\langle 2|\nabla j|^{2}\right\rangle_{\phi_{i}} .
$$

Since, on the other hand,

$$
\operatorname{Re}\left\langle H_{\eta}-\mu_{i}\right\rangle=\left\langle H^{0}-|\nabla \eta|^{2}-\mu_{i}\right\rangle,
$$

(10.8) follows.

Combining estimates (10.2) and (10.8) for $u=j e^{\eta} \phi_{i}$ yields

$$
b f^{\alpha / 2}\left\|j e^{\eta} \phi_{i}\right\|_{1} \leqq 2 \sup |\nabla j|\left\|\phi_{i}\right\| .
$$

Take now $\eta(x)=\varrho_{E^{\prime}, f}(x)$. Then, due to Proposition 3.1, it obeys (10.3). Moreover, a simple estimate on geodesic lengths shows

$$
\left|\varrho_{E^{\prime}, f}(x)-\varrho_{E_{0}, f}(x)\right| \leqq C f^{-1}\left|E^{\prime}-E_{0}\right|=C f^{\alpha-1} .
$$

On the other hand, $j$ can be chosen so that $\nabla j=O\left(f^{\beta}\right)$. The last three estimates imply (10.1).

\section{Exponential Decay of Resonance States}

Recall that in our terminology the resonance states are eigenfunctions of $H(f, \phi)$, $\phi>0$, corresponding to isolated (complex) eigenvalues $\left(E_{i}(f)\right)$. We obtain exponential bounds on these eigenfunctions in terms of the corresponding geodesic length (instanton action). These bounds, imply, in particular, that outside of the interior allowed region the resonance states decay exponentially as $f \rightarrow 0$. Our method is patterned on that of [S 2].

Let $\sigma_{E, f}$ be the distance between the origin and $S_{E, f}^{+}$in the geodesic metric $\varrho_{E, f}(x, y)$. Let

$$
d_{E, f}(x)=\min \left(\varrho_{E, f}(x), \sigma_{E, f}\right) .
$$

Let $\psi_{i}$ be an eigenfunction of $H(f, \phi)$ (resonance state) corresponding to an eigenvalue $E_{i}(f)$ splitting from $E_{0}$. The main result of this section is

Theorem 11.1. Let $\Omega_{R, f}^{(n)}$ be $\mathbb{R}^{v N}$ for $n=0$ and be the set $\left\{x \in \mathbb{R}^{v N}|| F \cdot x \mid \leqq R\right\}$ for $n>0$. Then

$$
\int_{\Omega_{R}, f} e^{2 d_{E_{0}, f}+O\left(f^{-\beta}\right)}\left|\nabla^{n} \psi_{i}\right|^{2} \leqq C_{R} f^{2 \beta-\alpha}
$$

for any $f$ sufficiently small, for any $R>0$, for $n=0,1,2$ and with $C_{R}$, independent of $f$.

Proof. Our proof is based on comparing eigenfunctions of $H(f, \phi)$ and $H_{f}^{0}$. Henceforth we omit the arguments (subindexes) $f$ and $\phi$. Let $P_{0}$ and $P$ be the 
eigenprojections of $H^{0}$ and $H$, respectively, on the spans of eigenspaces corresponding to the eigenvalues splitting from $E_{0}$. Let $\left\{\phi_{k}\right\}$ and $\left\{\eta_{k}\right\}$ be bases in $\operatorname{Ran} P_{0}$ and $\operatorname{Ran} P$, respectively. The $\phi_{k}$ 's are chosen to be real eigenfunctions of $H^{0}$ with the eigenvalues $\mu_{k}$. Due to (9.3), for $f$ sufficiently small, bases in $\operatorname{Ran} P$ and $\operatorname{Ran} P_{0}$ can be chosen so that

$$
\left\langle\bar{\eta}_{i}, \phi_{j}\right\rangle \rightarrow \delta_{i j} \quad \text { as } \quad f \rightarrow 0 .
$$

By stability estimate (6.4), one we can choose a contour $\Gamma \subset \varrho(H) \cap \varrho\left(H^{0}\right)$ of order $O\left(f^{v}\right)$ encircling $E_{0}$ and the eigenvalues of $H$ splitting from $E_{0}$. Then

$$
P_{0}=\frac{1}{2 \pi i} \oint_{\Gamma} R_{0} d z \quad \text { and } \quad P=\frac{1}{2 \pi i} \oint_{\Gamma} R d z
$$

where $R_{0}$ and $R$ are the resolvents of $H^{0}$ and $H$, respectively.

Let $h$ be a smooth cut-off function defined as: $0 \leqq h \leqq 1, h=1$ inside $S_{f / 4}$ and $=0$ outside $S_{f}$ and obeying $\nabla^{n} h=O\left(f^{n \beta}\right)$. We use the second resolvent equation

$$
R h=h R_{0}-R W R_{0},
$$

where, since $V^{0}=V$ on $\operatorname{supp} h$,

$$
W=\left[p^{2}, h\right] .
$$

Applying Eq. (11.4) to $\phi_{k}$ and integrating the result over $\Gamma$ we obtain

$$
P h \phi_{k}=h \phi_{k}+g_{k},
$$

where

$$
g_{k}=\frac{1}{2 \pi i} \oint_{\Gamma} R W \phi_{k} \frac{d z}{z-\mu_{k}} .
$$

By the definition, the geodesic distance from 0 to $S_{f}$,

$$
\varrho_{E_{0}, f}\left(S_{f}\right)=\sigma_{E_{0}, f}+O\left(f^{-\beta}\right) .
$$

Using that $\left|\mu_{k}-E_{0}\right|=o(|\Gamma|)$, using stability estimate (6.4), using the relation

$$
W=-2 \nabla h \cdot \nabla-\Delta h,
$$

and using exponential bounds (10.1) on $\phi_{k}$ and $\nabla \phi_{k}$ and inequalities $\alpha<v<\beta$, we obtain

$$
\left\|g_{k}\right\| \leqq C f^{\beta-\alpha / 2} e^{-\sigma_{E_{0}, f}+O\left(f^{-\beta}\right)} .
$$

Next, since $H(\lambda, \theta)^{*}=\bar{H}(\lambda, \theta)$ (complex conjugation), we have that

$$
P \phi_{k}=\sum_{i}\left\langle\bar{\eta}_{i}, \phi_{k}\right\rangle \eta_{i}
$$

Thus we can rewrite (11.5) as

$$
\sum_{i}\left\langle\bar{\eta}_{i}, h \phi_{k}\right\rangle \eta_{i}=h \phi_{k}+g_{k} .
$$

Due to (11.3) and the exponential bound on $(1-h) \phi_{k}$ which follows from (10.1) and the localization of $1-h$ we conclude that that matrix $\left[\left\langle\bar{\eta}_{j}, h \phi_{k}\right\rangle\right]$ is invertible for $f$ 
sufficiently small and its inverse bounded, say, by 2 . Then equations (11.8)-(11.9) and (10.1) yield bound (11.2) for $n=0$ (remember that $\psi_{k} \in \operatorname{Ran} P$ ).

Next, using the eigen-equation we derive the exponential bounds on $\psi_{i}$. In the rest of the proof we omit all subindices and denote by $\chi$ a smooth function vanishing outside a small neighbourhood of $\Omega^{(1)}$ and $=1$ on $\Omega^{(1)}$. We show that

$$
\left\|e^{d} \chi \Delta \psi\right\| \leqq \text { const }\left\|e^{d} \psi\right\|,
$$

where the constant is independent of $f$. Due to the eigen-equation on $\psi$ and the definition of $\chi$

$$
\left\|e^{d} \chi \Delta \psi\right\| \leqq C\left(\left\|\chi e^{d} V \psi\right\|+\left\|e^{d} \psi\right\|\right),
$$

where $V$ is the (deformed) total potential for $f=0$. Next we use the infinitesimal $\Delta$-boundedness of $V$ (the Kato inequality, see [CFKS]):

$$
\left\|\chi e^{d} V \psi\right\| \leqq \varepsilon\left\|\Delta\left(\chi e^{d} \psi\right)\right\|+C_{\varepsilon}\left\|e^{d} \psi\right\|
$$

valid for any $\varepsilon>0$. Now, we claim that

$$
\nabla^{n} d \text { is bounded on } \operatorname{supp} \chi \text { for } n=1,2 \text {. }
$$

Indeed, this follows from the fact that the geodesics avoid the incidence planes for the subsystems $B$ (i.e. where $x_{i}=x_{j}$ for some $i, j \in B$ ) ("the hiker looking for the fastest pass would not climb over the pick of the mountain"). Using (11.13), and using the Schwarz inequality integrating by parts, we obtain

$$
\left\|\chi e^{d} \nabla \psi\right\| \leqq \alpha\left\|\chi e^{d} \Delta \psi\right\|+C_{\alpha}\left\|e^{d} \psi\right\| .
$$

Taking into account (11.13) and (11.14) we arrive at

$$
\left\|\chi e^{d} V \psi\right\| \leqq \varepsilon\left\|\chi e^{d} \Delta \psi\right\|+C_{\varepsilon}\left\|e^{d} \psi\right\| .
$$

Combining this with (11.11), we obtain (11.10). Inequalities (11.2) for $n=0,(11.10)$ and (11.14) yield (11.2) for $n=0,1,2$.

\section{Width of Resonances. Proof of Theorem 6.3}

Let $\psi_{k}$ be the eigenfunctions corresponding to the eigenvalues $E_{k}(f)$. Let $\Omega_{f}$ be the interior of $S_{f / 2}$ and observe that, due to the fact that the deformation begins outside of $\Omega_{f}$ we have

$$
H(f, \phi)=H_{f}(\text { real }) \text { on } \Omega_{f} .
$$

Consider the eigenequation

$$
H(f, \phi) \psi_{k}=E_{k}(f) \psi_{k} .
$$

Multiplying it by $\bar{\psi}_{k}$ and integrating over $\Omega_{f}$ we obtain

$$
\int_{\Omega_{f}} \bar{\psi}_{k} H_{f} \psi_{k}=E_{k}(f) \int_{\Omega_{f}}\left|\psi_{k}\right|^{2}
$$

Applying Green's theorem to the kinetic energy term, we obtain

$$
E_{k}(f) \int_{\Omega_{f}}\left|\psi_{k}\right|^{2}=\int_{\Omega_{f}}\left|\nabla \psi_{k}\right|^{2}-\int_{\partial \Omega_{f}} \bar{\psi}_{k} \frac{\partial \psi_{k}}{\partial n}+\int_{\Omega_{f}} V_{f}\left|\psi_{k}\right|^{2},
$$


where $n$ is the normal vector to the surface $\partial \Omega_{f}$. Taking the imaginary part of this equation we arrive at

$$
\operatorname{Im} E_{k}(f)=\frac{\operatorname{Im} \int_{\partial \Omega_{f}} \psi_{k} \frac{\partial \psi_{k}}{\partial n}}{\int_{\Omega_{f}}\left|\psi_{k}\right|^{2}}
$$

\section{Lemma 12.1.}

$$
\int_{\partial \Omega_{f}}\left|\psi_{k} \nabla \psi_{k}\right| \leqq C f^{2 \beta-\alpha} e^{-2 \sigma_{E_{0}, f}+O\left(f^{-\beta}\right)} .
$$

Proof. We omit the subindices $E_{0}$ and $f$ at $\sigma$ and $d$. Denote

$$
g=e^{2 d} \nabla \psi_{k} \bar{\psi}_{k} .
$$

Then, due to the definition of $d$ we have

$$
\int_{\partial \Omega_{f}}\left|\psi_{k} \nabla \psi_{k}\right| \leqq e^{-2 \sigma} \int_{\partial \Omega_{f}}|g|
$$

Applying the Schwarz inequality, we obtain

$$
\int_{\partial \Omega_{f}}|g| \leqq\left[\int_{\partial \Omega_{f}} e^{2 d}\left|\nabla \psi_{k}\right|^{2}\right]^{1 / 2}\left[\int_{\partial \Omega_{f}} e^{2 d}\left|\psi_{k}\right|^{2}\right]^{1 / 2}
$$

Let $W_{f}$ be the layer between $S_{f}$ and $S_{f / a}, 2^{\beta}<a^{\beta}<2$. Let $\eta$ be a smooth function supported in $W_{f}$ and $=1$ on $\partial \Omega_{f}$. Since $\partial \Omega_{f}$ is a regular surface of the codimension 1, applying a Sobolev type embedding theorem to $e^{d} \eta u$, one obtains

$$
\int_{\partial \Omega_{f}} e^{2 d}|u|^{2} \leqq C\left(\int_{W_{f}} e^{2 d}|u|^{2}+\int_{W_{f}}\left|\nabla e^{d} u\right|^{2}\right),
$$

where $C$ is a numerical constant. Applying this inequality to $\psi_{k}$ and $\nabla \psi_{k}$, using the exponential bounds on $\nabla^{n} \psi_{k}$ with $n \leqq 2$ [see Eq. (11.2)], using $W_{f} \subset \Omega_{R, f}^{(1)}$ for $R$ sufficiently large and (11.13), we obtain

$$
\int_{\partial \Omega_{f}}|g| \leqq \text { const } f^{2 \beta-\alpha} .
$$

Next, due to (11.2), for $f$ sufficiently small,

$$
\int_{\Omega_{f}}\left|\psi_{k}\right|^{2} \geqq 2 / 3 \text {. }
$$

The last three relations imply (6.6).

In the method above we have followed $[\mathrm{S} 2]$ (see $[\mathrm{Av}, \mathrm{HS}]$ for earlier versions).

\section{Stability Estimate}

In this section we prove Theorem 6.1 which gives a bound on the resolvent of $H(f, \phi)$ which plays a crucial role in the stability result (Sect. 9). This is technically the most demanding section of this paper. One should consult Sect. 6 for notation. We estimate $H(f, \phi)$ separately on different regions of the configuration space and then patch the obtained estimates together. 
Proposition 13.1. Let $u \in C_{0}^{\infty}$ and be supported in the exterior of $S_{2 f}$. Then

$$
-\operatorname{Im}\langle H(f, \phi)\rangle_{u} \geqq \frac{1}{4} \phi f^{1-\gamma}\|u\|^{2} .
$$

Proof. Recall that $\chi_{f}=1$ on the exterior of $S_{2 f}$. We introduce the domains

$$
\Lambda_{i, f}=\left\{x \in \mathbb{R}^{v N} \mid x_{i} \cdot e \geqq f^{-\beta}\right\} \text {. }
$$

Clearly $\Lambda_{i, f}, i=1, \ldots, N$, cover the exterior of $S_{2 f}$. Let $\left\{j_{i, f}\right\}$ be an associated partition of unity:

$$
\begin{aligned}
\sum j_{i, f}^{2}=1 & \text { on the exterior of } S_{2 f}, \\
& \operatorname{supp} j_{i, f} \subset A_{i, f}, \\
& \left|\nabla^{n} j_{i, f}\right| \leqq C f^{n \beta} .
\end{aligned}
$$

A variant of the IMS localization formula with $\left\{j_{i, f}\right\}$ (see e.g. [CFKS]) and Eq. (13.5) yield

$$
\operatorname{Im}\langle H(f, \phi)\rangle_{u} \leqq \Sigma \operatorname{Im}\langle H(f, \phi)\rangle_{u_{\imath}}+C f^{2 \beta}\|u\|^{2},
$$

where $u_{i}=j_{i, f} u$.

Next, we estimate each term under the sum on the right-hand side of (13.6). We begin with the kinetic energy term (recall $\chi_{f} \equiv 1$ now)

$$
T_{\phi}=\Sigma p_{i, \phi}^{2} \text { on the exterior of } S_{2 f},
$$

where $p_{i, \phi}^{2}$ is the deformation of $p_{i}^{2}$ by the vector field $v_{i} e$. Let

$$
v_{i}^{(n)}=w^{(n)}\left(x_{i} \cdot e\right) .
$$

Since

$$
v_{i}^{(n)}=O\left(f^{-\gamma+n \beta}\right),
$$

we have due to Lemma A.1 given in the appendix that

$$
-\operatorname{Im} p_{i, \phi}^{2} \geqq \phi q_{i} v_{i}^{\prime} q_{i}^{\prime}-C f^{2 \beta},
$$

where $q_{i}=e \cdot p_{i}$.

Now we proceed to the potentials:

$$
V_{j, f}(\phi)=V_{j}\left(x_{j}+i \phi v_{j} e\right)-f\left(x_{j} \cdot e+i e v_{j}\right) .
$$

Using the mean value theorem, we write this as

$$
V_{j, f}(\phi)=V_{j . f}-i \phi f v_{j}\left(1-I_{j}\right),
$$

where (with $0 \leqq \bar{\phi} \leqq \phi$ )

$$
I_{j}=f^{-1} \frac{\partial V_{j}}{\partial e}\left(x_{j}+i \bar{\phi} v_{j} e\right)
$$

Since

$$
x_{j} \cdot e \geqq \operatorname{const} f^{-\beta} \quad \text { on } \operatorname{supp} v_{j},
$$

we have due to condition (III) that

$$
I_{j}=O\left(f^{\varepsilon_{1}}\right) \quad \text { on } \operatorname{supp} v_{j},
$$


with $\varepsilon_{1}=(1+\mu) \beta-1>0$. Hence

$$
-\operatorname{Im} V_{j, f}(\phi) \geqq f \phi v_{j}\left(1-C f^{\varepsilon_{1}}\right) \geqq 0 .
$$

Next, we consider $V_{i j}(\phi)$. Write

$$
V_{i j}(\phi)=V_{i j}(\zeta, u)
$$

where $u$ is the projection of $x_{i}-x_{j}$ onto $e^{\perp}$ and

$$
\zeta=\left(x_{i}-x_{j}\right) \cdot e+i \phi\left(v_{i}-v_{j}\right) \text {. }
$$

Compute

$$
\operatorname{Re} \zeta=x_{i} \cdot e-x_{j} \cdot e \text { and } \operatorname{Im} \zeta=\phi\left(v_{i}-v_{j}\right) .
$$

Since $w(s)$ is monotonically non-decreasing, we have

$$
\operatorname{Re} \zeta \operatorname{Im} \zeta \geqq 0 \text {. }
$$

Hence, due to condition (IV) on $V_{i j}$,

$$
\operatorname{Im} V_{i j}(\phi) \leqq 0 .
$$

Now combining Eqs. (13.9), (13.14), and (13.19) together with (5.11) we obtain for $u \in C_{0}^{\infty}\left(\Lambda_{j, f}\right)$,

$$
-\operatorname{Im}\langle H(f, \phi)\rangle_{u} \geqq \Sigma\left(\phi\left\langle q_{i} v_{i}^{\prime} q_{i}\right\rangle_{u}+\frac{1}{2} \phi f\left\langle v_{i}\right\rangle_{u}\right)-C f^{2 \beta}\|u\|^{2} .
$$

Since $v_{j} \geqq 0$ for all $j$ and $v_{i} \geqq f^{-\gamma}$ on $\Lambda_{i, f}$, we arrive at (13.1).

Now we proceed to the next region.

Proposition 13.2. Let $u \in C_{0}^{\infty}$ be supported in $K_{E^{\prime}, f}$. Then there is $\delta_{1}>0$ depending only on the potentials such that

$$
\operatorname{Re}\langle H(f, \phi)-z\rangle \geqq \delta_{1} f^{\alpha}\|u\|_{1}^{2}
$$

for all u's, all $z$ in the disc $|z|<f^{v}$ and for all $f$ sufficiently small.

Proof. The proposition follows from representation (4.1) of $K_{E^{\prime}, f}$, inequality $\alpha<\varepsilon$ [see (6.3)] and Lemma 13.3 below taken with $\lambda=\frac{1}{2 c} f^{\alpha}$.

Lemma 13.3. There is $c>0$ depending only on the potentials such that

$$
\operatorname{Re}\langle H(f, \phi)\rangle \geqq\left\langle W_{a(x), f}(x)\right\rangle+\lambda\|p u\|^{2}-\left(\lambda c+C f^{\varepsilon}\right)\|u\|^{2}
$$

for any $0 \leqq \lambda \leqq 1$, any $u \in C_{0}^{\infty}\left(B_{f}\right)$ and any $f$ sufficiently small.

Proof. It follows from the definition of $\Omega_{a, f}$ that on $\Omega_{a, f}$

$$
I_{a}(\phi)=O\left(f^{\mu \beta}\right) .
$$

Furthermore, Eq. (13.10) and (13.13) imply that

$$
V_{j, f}(\phi)=V_{j, f}-i \phi f v_{j}+O\left(f^{\varepsilon_{1}}\right) .
$$


Next, we show that

$$
V_{i j}(\phi)=V_{i j}+\phi f^{\beta-\gamma} O_{1}(1)
$$

where $O_{1}(1)$ is a $\Delta$-bounded operator with the relative bound 0 , uniformly in $\phi$ and $f$. Indeed, by the mean-value theorem,

$$
V_{i j}(\phi)=V_{i j}+i \phi f^{-\gamma} v_{i j} \frac{\partial V_{i j}}{\partial e}\left(x_{i j}+i \bar{\phi} v_{i j} e\right)
$$

for some $0 \leqq \bar{\phi} \leqq \phi$, where we use notation:

$$
x_{i j}=x_{i}-x_{j} \text { and } v_{i j}=v_{i}-v_{j} .
$$

Applying the mean-value theorem again yields:

$$
v_{i j}=O\left(x_{i j} \cdot e f^{\beta}\right) .
$$

Using condition (II) on $V_{i j}$ we arrive at the conclusion (13.25).

Turn to the kinetic energy term. Lemma A.1 of Appendix implies that

$$
\operatorname{Re}\left(p_{B, \phi}\right) \geqq \frac{1}{4} p_{B}^{2}-C \phi f^{2 \beta}
$$

for $\phi$ sufficiently small and with $C$ independent of $\phi$ and $f p_{B}^{2}=\sum_{B} p_{i}^{2}$.

Collecting estimates (10.5), (13.23)-(13.25), (13.29) and using decomposition formula (7.8), we obtain

$$
\operatorname{Re}\langle H(f, \phi)\rangle \geqq\left\langle W_{a, f}\right\rangle+\lambda\|p u\|^{2}-\left(\lambda c+C f^{\varepsilon}\right)\|u\|^{2}
$$

for any $u \in C_{0}^{\infty}$ supported in $\Omega_{a, f}$.

Now we patch together the estimates given in Eq. (13.30). To this end we use a partition of unity $\left\{j_{a, f}\right\}$ introduced in the proof of Corollary 7.3. Applying to $H(f, \phi)$ the IMS localization formula with this partition yields:

$$
\operatorname{Re}\langle H(f, \phi)\rangle_{u} \geqq \Sigma \operatorname{Re}\langle H(f, \phi)\rangle_{a}-C f^{2 \beta}\|u\|^{2},
$$

where the subindex $a$ stands for the mean with the function $u_{a}=j_{a, f} u$. Taking into account (7.17) and applying inequalities (13.30) yields

$$
\operatorname{Re}\langle H(f, \phi)\rangle \geqq \sum \lambda\left\|p u_{a}\right\|^{2}-\left(\lambda c+C f^{\varepsilon}\right)\|u\|^{2}+\left\langle W_{a, f}\right\rangle_{a} .
$$

Using again the IMS localization formula but this time for $p^{2}$ and using (3.7) and (7.1), we arrive at (13.22).

Now we put together the local estimates on $H(f, \phi)$ obtained above. Let $\left\{\chi_{i}\right\}_{1}^{3}$, be a partition of unity normalized as

$$
\sum \chi_{i}^{2}=1
$$

and having the properties

$$
\begin{gathered}
\chi_{1} \text { is supported in the exterior of } S_{2 f}, \\
\chi_{2} \text { is supported on } K_{E^{\prime} . f}, \\
\chi_{3} \text { is supported in } B_{f / 2}^{c},
\end{gathered}
$$


and

$$
\nabla \chi_{i}=O\left(f^{n \beta}\right) .
$$

We proceed to the estimates. Using that

$$
\sum \chi_{i}^{4} \leqq 1,
$$

we obtain by sticking the partition inside of the norm

$$
\|(H(f, \phi)-z) u\|^{2} \geqq \Sigma\left\|\chi_{i}^{2}(H(f, \phi)-z) u\right\|^{2} .
$$

Commuting $\chi_{i}$ to the right we continue

$$
\|(H(f, \phi)-z) u\|^{2} \geqq \sum_{i=1}^{3}\left\|\chi_{i}(H(f, \phi)-z) \chi_{i} u\right\|^{2}-R,
$$

where

$$
R=\sum_{i=1}^{3}\left\|\chi_{i}\left[\chi_{i}, T_{\phi}\right] u\right\|^{2}
$$

Rearranging the commutator on the right-hand side, we obtain

$$
R \leqq \frac{1}{2} \sum_{i=1}^{3}\left\|\left(\chi_{i}\left[\chi_{i}, T_{\phi}\right]+\left[\chi_{i}, T_{\phi}\right] \chi_{i}\right) u\right\|^{2}+\frac{1}{2}\left\|\left[\chi_{i},\left[\chi_{i}, T_{\phi}\right]\right] u\right\|^{2}
$$

By (13.37) and (5.12),

$$
\Sigma\left\|\left[\chi_{i},\left[\chi_{i}, T_{\phi}\right]\right] u\right\|^{2} \leqq C f^{4 \beta}\|u\|^{2}
$$

By (13.33),

$$
\sum_{i=1}^{3}\left(\chi_{i}\left[\chi_{i}, T_{\phi}\right]+\left[\chi_{i}, T_{\phi}\right] \chi_{i}\right)=0 .
$$

Thus we can exclude the first summand in the first term on the left-hand side of (13.42). This leads to

$$
R \leqq 2 \sum_{i=2}^{3}\left\|\left[\chi_{i}, T_{\phi}\right] \chi_{i} u\right\|^{2}+C f^{4}\|u\|^{2}
$$

Computing the commutator $\left[\chi_{i}, T_{\phi}\right]$ and using (13.37), we obtain

$$
R \leqq C f^{2 \beta} \sum_{i=2}^{3}\left\|p \chi_{i} u\right\|^{2}+C f^{4 \beta}\|u\|^{2} .
$$

Now we estimate the first sum on the right-hand side of (13.40). The Schwarz inequality yields

$$
\left\|\chi_{1}(H(f, \phi)-z) \chi_{1} u\right\|\|u\| \geqq-\operatorname{Im}\langle H(f, \phi)-z\rangle_{\chi_{1} u} .
$$

Using inequality (13.1) and the condition

$$
\operatorname{Im} z \geqq-f^{\nu} \quad \text { and } \quad v>1-\gamma,
$$

we obtain

$$
\left\|\chi_{1}(H(f, \phi)-z) \chi_{1} u\right\|\|u\| \geqq \frac{1}{8} \phi f^{1-\gamma}\left\|\chi_{1} u\right\|^{2} .
$$


Furthermore using the inequality

$$
x^{2} \geqq 2 a x y-a^{2} y^{2}
$$

valid for any $a>0$, with $a=f^{2 v-(1-\gamma)}$, we conclude

$$
\left\|\chi_{1}(H(f, \phi)-z) \chi_{1} u\right\|^{2} \geqq \frac{1}{4} \phi f^{2 v}\left\|\chi_{1} u\right\|^{2}-f^{2 \sigma}\|u\|^{2},
$$

where

$$
\sigma=2 v-(1-\gamma)>v .
$$

Next, we apply the Schwarz inequality again

$$
\left\|\chi_{2}(H(f, \phi)-z) \chi_{2} u\right\|\|u\| \geqq \operatorname{Re}\langle H(f, \phi)-z\rangle_{\chi_{2} u} .
$$

Using inequalities (13.21) and (13.50) with $a=f^{2 v-\alpha}$ and using that $2 v-\alpha>2 v$ $-(1-\gamma)=\sigma$, we arrive at

$$
\left\|\chi_{2}(H(f, \phi)-z) \chi_{2} u\right\|^{2} \geqq \frac{1}{2} \delta_{1} f^{2 v}\left\|\chi_{2} u\right\|_{1}^{2}-f^{2 \sigma}\|u\|^{2} .
$$

Finally, commuting $\chi_{3}$ to the right and using (13.37) and that $H(f, \phi)=H+O(f)$ on $\operatorname{supp} \chi_{3}$, we get

$$
\left\|\chi_{3}(H(f, \phi)-z) \chi_{3} u\right\| \geqq\left\|(H-z) \chi_{3}^{2} u\right\|-C f^{\beta}\left\|p \chi_{3} u\right\|-C f^{2 \beta}\left\|\chi_{3} u\right\| .
$$

Since for $z$ in the annulus (6.1), the distance to the spectrum of $H$ is $\geqq \frac{1}{2} f^{\nu}$, the spectral theorem yields

$$
\left\|\chi_{3}(H(f, \phi)-z) \chi_{3} u\right\| \geqq \frac{1}{4} \delta_{2} f^{v}\left\|\chi_{3}^{2} u\right\|_{2}-C f^{\beta}\left\|p \chi_{3} u\right\|
$$

for $f$ sufficiently small. Here we have used that $2 \beta>\sigma$. Next, the Schwarz inequality gives

$$
\left\|p \chi_{3} u\right\|^{2} \leqq\|u\|\left\|\chi_{3} p^{2} \chi_{3} u\right\|
$$

Commuting $\chi_{3}$ on the right-hand side to the right, evaluating a simple commutator and using (13.37), we get

$$
\left\|p \chi_{3} u\right\|^{2} \leqq\|u\|\left(\left\|p^{2} \chi_{3}^{2} u\right\|+f^{\beta}\left\|p \chi_{3} u\right\|\right)+f^{2 \beta}\|u\|^{2} .
$$

This inequality with $f$ sufficiently small implies that for any $\varepsilon>0$,

$$
\frac{3}{2} \sqrt{\varepsilon}\left\|p \chi_{3} u\right\|-\varepsilon\|u\| \leqq\left\|p^{2} \chi_{3}^{2} u\right\| .
$$

Combining this with (13.55) and using that $\beta>v$ we arrive at the estimate

$$
\left\|\chi_{3}(H(f, \phi)-z) \chi_{3} u\right\| \geqq \frac{1}{4} \delta_{2} \sqrt{\varepsilon} f^{v}\left\|\chi_{3} u\right\|_{1}-\frac{1}{4} \delta_{2} \varepsilon f^{v}\|u\|,
$$

provided $f$ is sufficiently small. Collecting estimates (13.40), (13.46), (13.51), (13.53), (13.59), using that $\sigma>v$ and taking $\varepsilon$ appropriately small:

$$
\delta_{2} \varepsilon \leqq \frac{1}{3} \min \left(\delta_{2} \sqrt{\varepsilon}, \sqrt{\delta_{1}}, \sqrt{\phi}\right) \equiv \delta,
$$

we arrive at (6.4) with $\xi \leqq\left(\sum_{i=2}^{3} \chi_{i}^{2}\right)^{1 / 2}$, provided $f$ is sufficiently small. 


\section{Appendix}

In this appendix we compute explicitly the distorted kinetic energy term. Since we are interested in the exterior of $S_{2 f}$ we can set $\chi_{f}=1$ in (5.9). Thus due to the form of the vector field $v$ the computation reduces to the one-dimensional case. Namely, on the exterior of $S_{2 f}$

$$
p_{i, \phi}=\left(\left(1+i u\left(x_{i} \cdot e\right)\right)^{-1} e \cdot p_{i}, p_{i}^{\perp}\right),
$$

where $p_{i}^{\perp}$ is the projection of $p_{i}$ onto the plane $e^{\perp}$ and $u(s)=\phi w(s)$ [see (13.7) for the relation between $T_{\phi}$ and $p_{i, \phi}$ and for the definition of $\left.p_{i, \phi}\right]$. Let

$$
p_{u}=(1+i u)^{-1} p
$$

where $p=-i \frac{\partial}{\partial x}, u=u(x)$, and $x \in \mathbb{R}^{1}$. A simple computation yields

$$
p_{u}^{2}=p(1+i u)^{-2} p+u^{\prime}(1+i u)^{-3} p .
$$

Taking the real and imaginary parts of this expression, we obtain further

$$
\operatorname{Re} p_{u}^{2}=p \frac{1-u^{2}}{\left(1+u^{2}\right)^{2}} p+\operatorname{Re}\left(u^{\prime} \frac{1-3 u^{2}}{\left(1+u^{2}\right)^{3}} p\right)+\left(u^{\prime} u \frac{3-u^{2}}{\left(1+u^{2}\right)^{3}}\right)^{\prime}
$$

and

$$
\operatorname{Im} p_{u}^{2}=-2 p u p-\operatorname{Re}\left(u^{\prime} u \frac{3-u^{2}}{\left(1+u^{2}\right)^{3}} p\right)+\left(u^{\prime} \frac{1-3 u^{2}}{\left(1+u^{2}\right)^{3}}\right)^{\prime} .
$$

\section{Lemma A.1.}

$$
-\operatorname{Im} p_{u}^{2} \geqq p u p-g_{1}
$$

where

$$
g_{1}=\frac{1}{4} \frac{\left(1-u^{2}\right)^{2}}{\left(1+u^{2}\right)^{6}} u^{\prime 2} u-\frac{1}{2}\left(u^{\prime} \frac{1-3 u^{2}}{\left(1+u^{2}\right)^{3}}\right)^{\prime}
$$

and

$$
\operatorname{Re} p_{u}^{2} \geqq \frac{1}{2} p\left(1+u^{2}\right)^{-2} p+g_{2}
$$

where

$$
g_{2}=\frac{1}{2}\left(u^{\prime} \frac{1+3 u^{2}}{\left(1+u^{2}\right)^{2}}\right)^{2}+\frac{1}{2}\left(u u^{\prime} \frac{3-u^{2}}{\left(1+u^{2}\right)^{3}}\right)^{\prime} .
$$

Proof. We demonstrate only the first estimate. We have

$$
\begin{aligned}
\left|\left\langle\frac{3-u^{2}}{\left(1+u^{2}\right)^{3}} u^{\prime} u p\right\rangle_{\psi}\right| & \leqq\left\|\frac{3-u^{2}}{\left(1+u^{2}\right)^{3}} u^{\prime} u^{1 / 2} \psi\right\|\left\|u^{1 / 2} p \psi\right\| \\
& \leqq \frac{1}{2 \alpha}\left\|\frac{3-u^{2}}{\left(1+u^{2}\right)^{3}} u^{\prime} u^{1 / 2} \psi\right\|^{2}+\frac{\alpha}{2}\langle p u p\rangle_{\psi},
\end{aligned}
$$

which implies estimate (A.5).

Acknowledgement. The author is grateful to W. Hunziker for many valuable discussions. 


\section{References}

[AC] Aguilar, J., Combes, J.M.: A class of analytic perturbations for one-body Schrödinger Hamiltonians. Commun. Math. Phys. 22, 269-275 (1971)

[Ag] Agmon, S.: Lectures on exponential decay of second-order elliptic equations. Princeton Math. Notes 29 (1982)

[Av] Avron, J.: Bender-Wu formulas for the Zeeman effect in hydrogen. Ann. Phys. 131, 73-94 (1981)

[AH] Avron, J.E., Herbst, I.W.: Spectral and scattering theory of Schrödinger operators related to the Stark effect. Commun. Math. Phys. 52, 239-254 (1977)

[BC] Balslev, E., Combes, J.M.: Spectral properties of Schrödinger Hamiltonians with dilation analytic potentials. Commun. Math. Phys. 22, 280-294 (1971)

[BCD] Briet, Ph., Combes, J.M., Duclos, P.: On the location of resonances for Schrödinger operators in the semi-classical limit. III. The shape resonances (in preparation)

[Bo] Bohm, A.: Quantum mechanics. Berlin, Heidelberg, New York: Springer 1986

[CDKS] Combes, J.M., Duclos, P., Klein, M., Seiler, R.: The shape resonances. Commun. Math. Phys. 110, 215-236 (1987)

[CFKS] Cycon, H.L., Froese, R., Kirsch, W., Simon, B.: Schrödinger operators. Berlin, Heidelberg, New York: Springer 1987

[Cyc] Cycon, H.L.: Resonances defined by modified dilations. Helv. Phys. Acta 53, 969-981 (1985)

[HaSi] Harrell II, E.M., Simon, B.: Duke Math. J. 47, 845-902 (1980)

[HeSi] Herbst, I.W., Simon, B.: Dilation analyticity in constant electric field. II. $N$-body problem, Borel summability. Commun. Math. Phys. 80, 181-216 (1981)

[HisSig 1] Hislop, P., Sigal, I.M.: Shape Resonances in Quantum Mechanics, in "Proceedings of the Intern. Conf. on Diff. Equations and Math. Physics, Knowles, I. (ed.) 1986; and Quantum Resonances, Memoir AMS (1987) (to appear)

[HisSig 2] Hislop, P., Sigal, I.M.: Lectures on spectral theory (in preparation)

[HS] Helffer, B., Sjöstrand, J.: Resonances en limite semi-classique, Mém. de la S.M.F. no. 24/25, Suppl. de la S.M.F. Bulletin 114 (1986)

[Hu 1] Hunziker, W.: Distortion analyticity and molecular resonance curves. Ann. Inst. H. Poincaré 45, 339-358 (1986)

[Hu 2] Hunziker, W.: Notes on asymptotic perturbation theory.... Helv. Phys. Acta 61, 257-304 (1988)

[k] Kato, T.: Perturbation theory for linear operators. Berlin, Heidelberg, New York: Springer 1966

[LL] Landau, L., Lifshitz, E.: Quantum mechanics, London: Pergamon Press 1965

[Mo] Morrey, Jr., C.B.: Multiple integrals in the calculus of variations. Berlin, Heidelberg, New York: Springer 1966

[Op] Oppenheimer, R.: Phys. Rev. 31, 66-81 (1928)

[S 1] Sigal, I.M.: Complex transformation method and resonances in one-body quantum systems. Ann. Inst. H. Poincaré 41, 103-114 (1984); and Addendum 41, 333 (1984)

[S 2] Sigal, I.M.: Sharp exponential bounds on resonance states and width of resonances. Adv. Appl. Math. 9, 127-166 (1988)

[S 3] Sigal, I.M.: Stark effect in multielectron systems: non-existence of bound states. Preprint, Toronto. Commun. Math. Phys. (to appear)

[S 4] Sigal, I.M.: Life-time of Stark resonances. In: Proc. of Conf. on Field Theory and Related Topics. Feldman, J., Rosen, L. (eds.) CMS Conference Proceedings 9, 233-246 (1988)

[S 5] Sigal, I.M.: Geometric parametrics and the many-body Birman-Schwinger principle. Duke Math. J. 50, 134-153 (1983)

[Sim 1] Simon, B.: The theory of resonances for dilation-analytic potentials... Ann. Math. 97, 246-274 (1973)

[Sim 2] Simon, B.: The definition of molecular resonance curves by the method of exterior complex scaling. Phys. Letts. 71A, 211-214 (1979) 
[Sim 3] Simon, B.: Semiclassical Analysis of low lying eigenvalues. II. Tunneling. Ann. Math. 120, 89-118 (1984)

[T] Titchmarsh, E.C.: Eigenfunction expansions associated with second order differential equations. Oxford: Oxford University Press 1958

[VH] Vock, E., Hunziker, W.: Stability of Schrödinger eigenvalue problems. Commun. Math. Phys. 83, 281-302 (1982)

Communicated by B. Simon

Received February 15, 1988 